\title{
Molecular Resolution of the Water Interface at an Alkali Halide with Terraces and Steps
}

Fumiaki Ito $^{1}$, Kei Kobayashi ${ }^{1,2}$, Peter Spijker ${ }^{3}$, Lidija Zivanovic ${ }^{3}$, Kenichi Umeda ${ }^{1}$, Tarmo Nurmi ${ }^{4}$, Nico Holmberg ${ }^{4}$, Kari Laasonen ${ }^{4}$, Adam S. Foster ${ }^{3,5}$, and Hirofumi Yamada ${ }^{1}$

${ }^{1}$ Department of Electronic Science and Engineering, Kyoto University, Kyoto 615-8510, Japan

${ }^{2}$ The Hakubi Center for Advanced Research, Kyoto University, Kyoto 615-8520, Japan

${ }^{3}$ COMP Centre of Excellence, Department of Applied Physics, Aalto University, Helsinki FI-00076, Finland ${ }^{4}$ Department of Chemistry, Aalto University, Helsinki FI-00076, Finland

${ }^{5}$ Division of Electrical Engineering and Computer Science, Kanazawa University, Kanazawa 920-1192, Japan

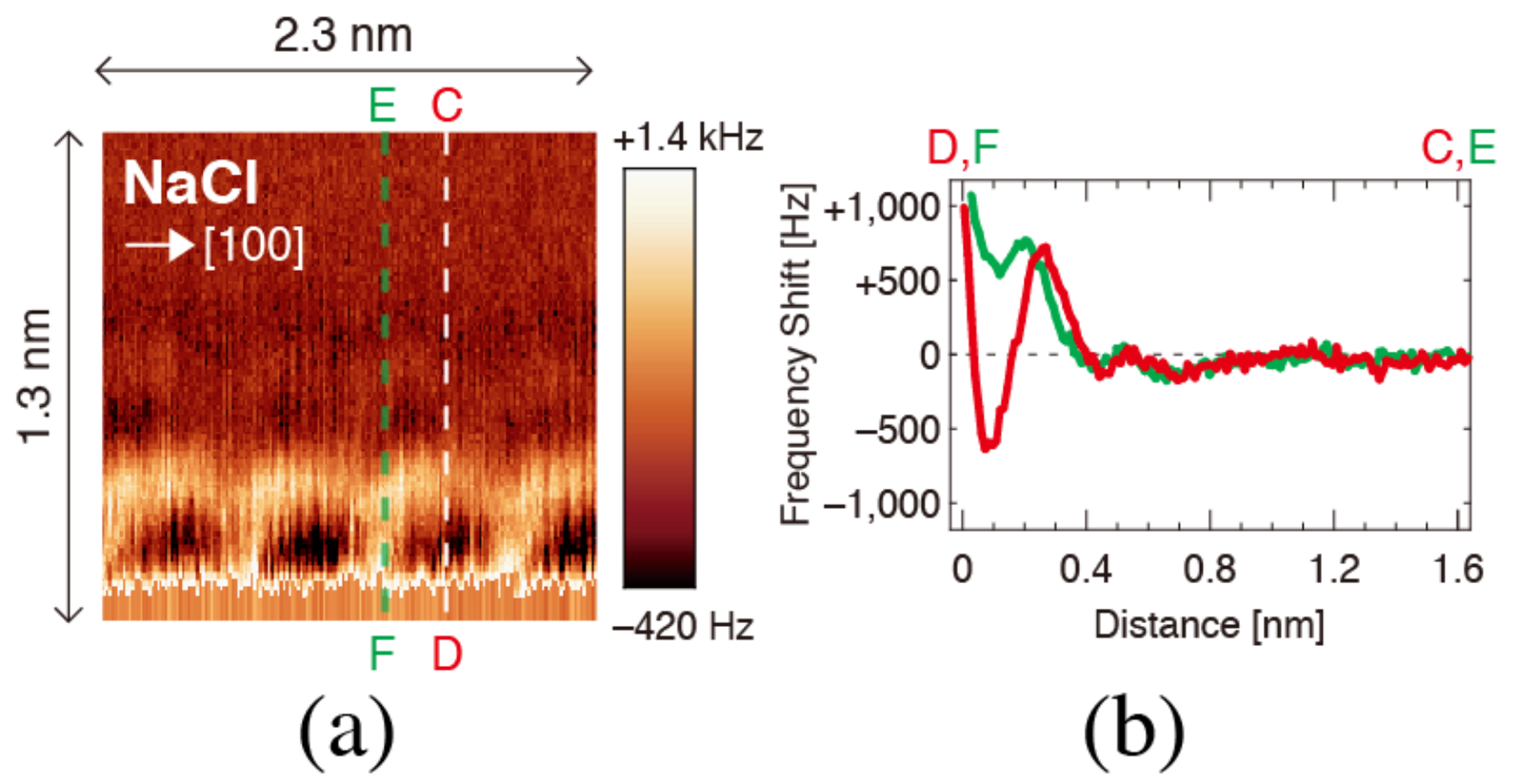

Figure S1: (a) 2D frequency shift map of a plane perpendicular to the $\mathrm{NaCl}(001)$ surface along the [001] direction $\left(A_{\mathrm{p}-\mathrm{p}}=0.25 \mathrm{~nm}, \Delta f_{\mathrm{th}}=2 \mathrm{kHz}\right)$. (b) Frequency shift versus distance curves along the lines $\mathrm{C}-\mathrm{D}$ and E-F indicated in (a). 


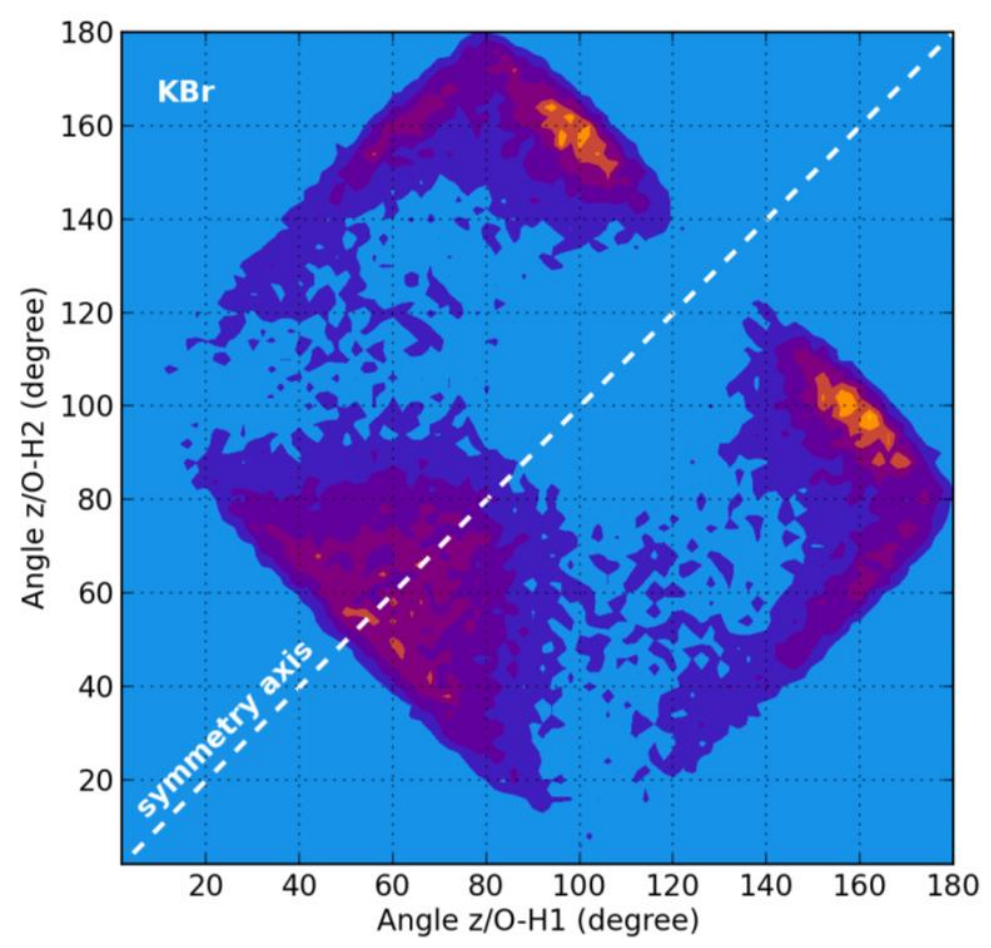

(a)



(b)

Figure S2: 2D correlation map of the angles of either $\mathrm{OH}$-bond of the water molecules with respect to the surface normal. As both $\mathrm{OH}$-bonds are indistinguishable there is a symmetry around the diagonal. The lighter the color the stronger the correlation. The two peaks at $(100,160)$ and $(160,100)$ are for waters above the anions, the single peak around $(60,60)$ for the water above the cations. In (a) the correlation for $\mathrm{KBr}$ is shown and in (b) for $\mathrm{NaCl}$. 

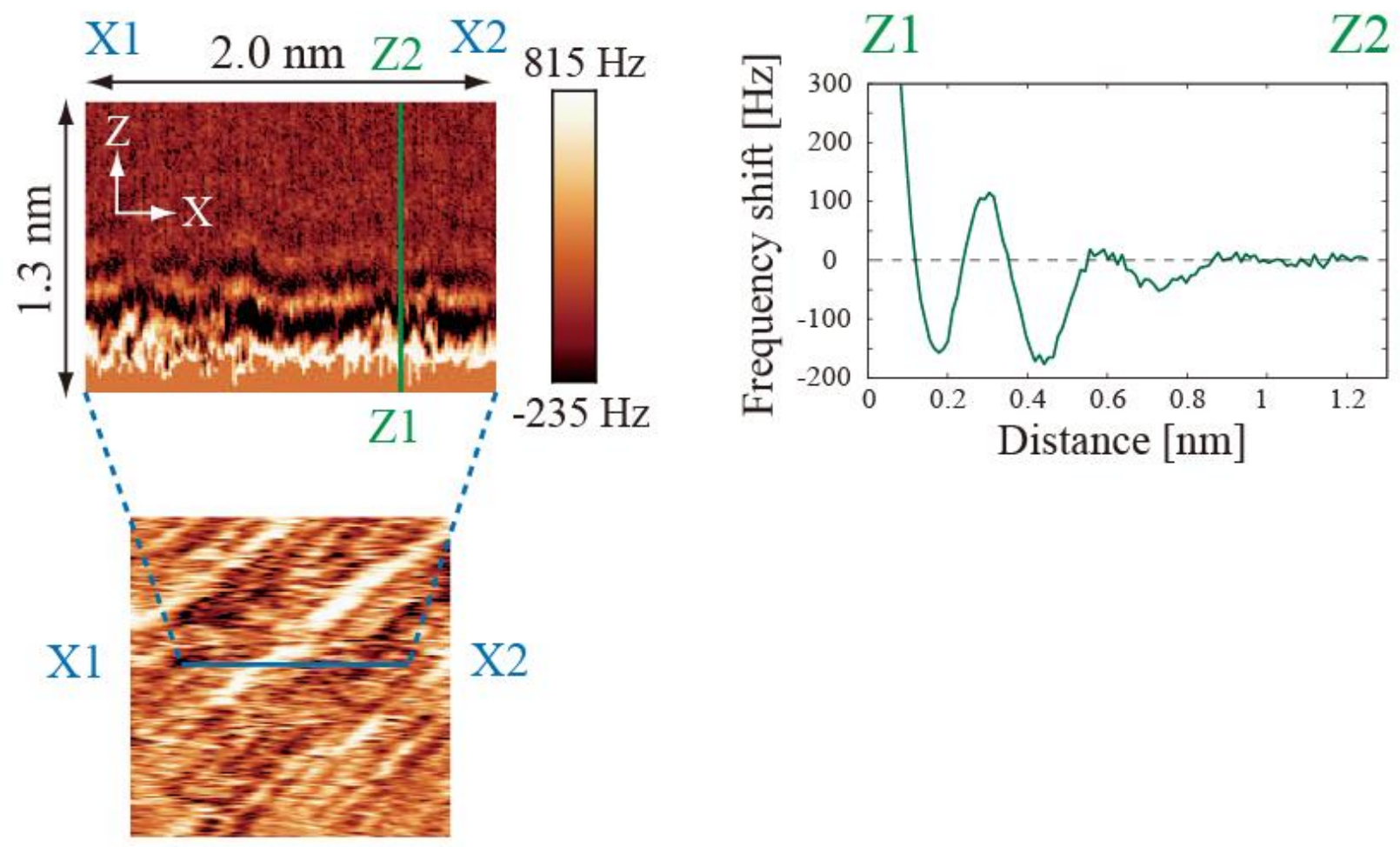

Figure S3: FM-AFM topographic image, 2D frequency shift map on a plane along the line X1-X2, and a frequency shift curve along the line $\mathrm{Z} 1-\mathrm{Z} 2$, on the $\mathrm{NaF}(001)$ surface.
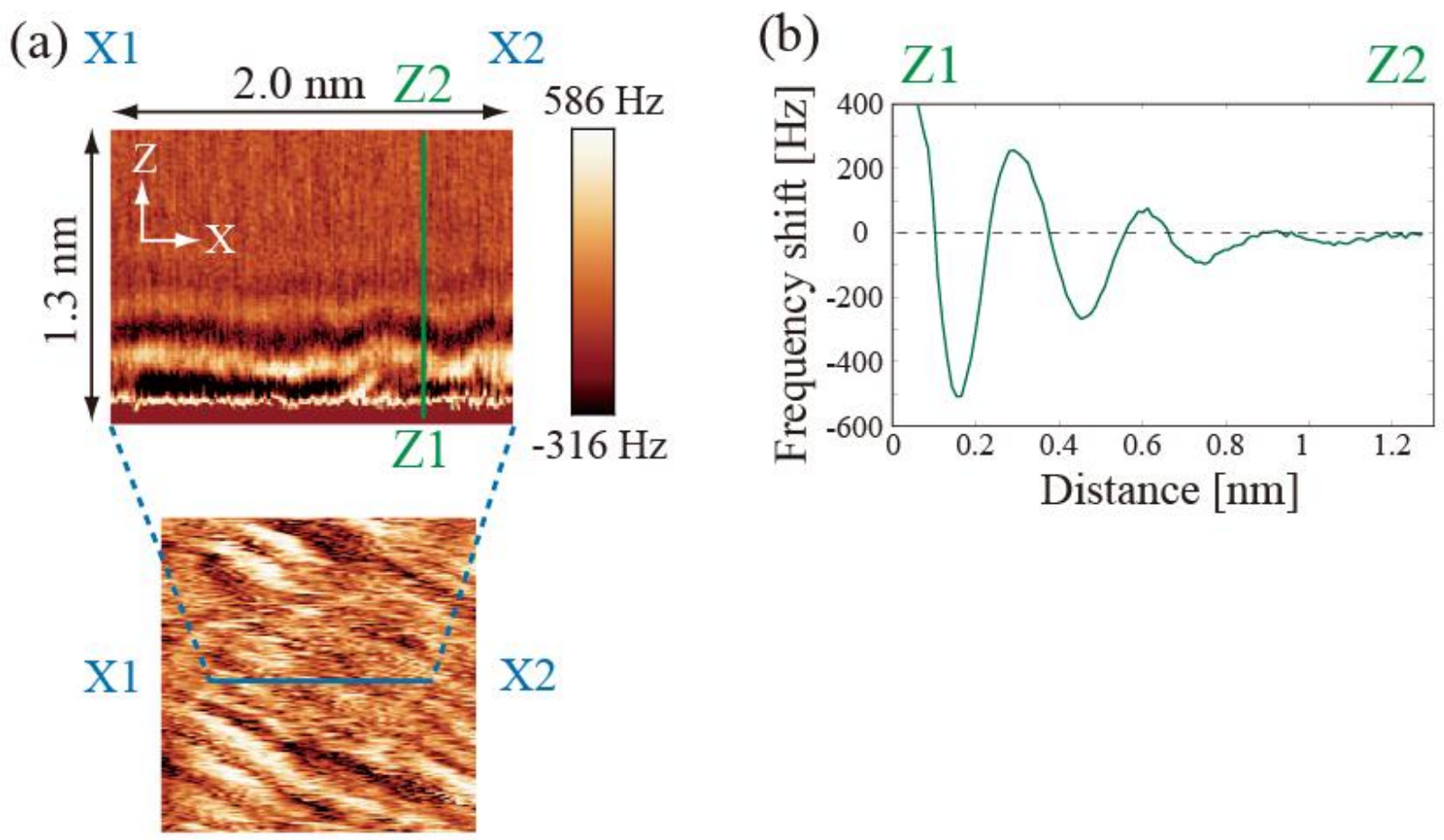

Figure S4: FM-AFM topographic image, 2D frequency shift map on a plane along the line X1-X2, and a frequency shift curve along the line $\mathrm{Z} 1-\mathrm{Z} 2$, on the $\mathrm{LiF}(001)$ surface. 

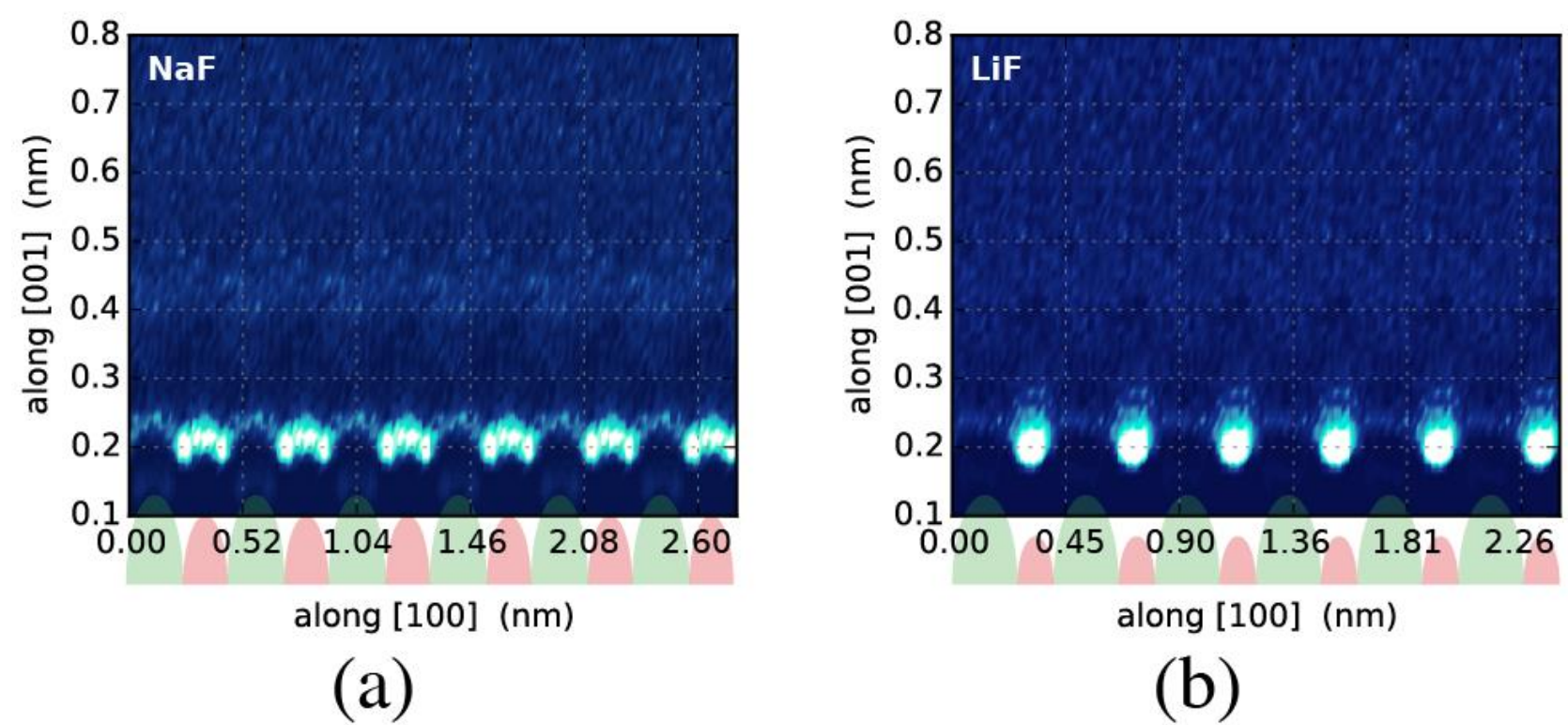

Figure S5: (a) 2D water density distribution in a plane along the [100] direction on the $\mathrm{NaF}(001)$ surface. (b) 2D water density distribution in a plane along the [100] direction on the $\operatorname{LiF}(001)$ surface.

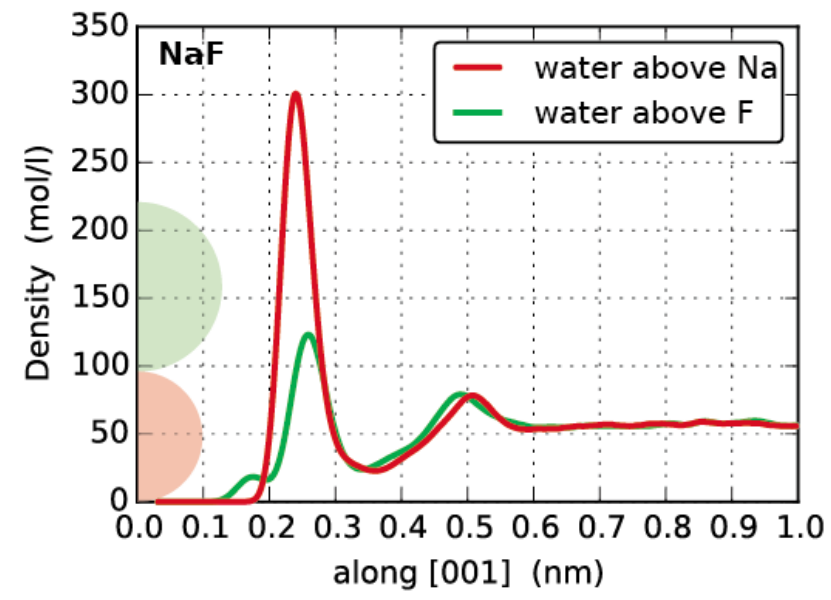

(a)



(b)

Figure S6: (a) 1D water density profile on $\mathrm{Na}^{+}$(red) and $\mathrm{F}^{-}$(green) ion as a function from the surface. Each curve presents the density of the water molecules on the half unit cell area $(0.23 \mathrm{~nm} \times$ $0.23 \mathrm{~nm}$ ). (b) $1 \mathrm{D}$ water density profile on $\mathrm{Li}^{+}$(red) and $\mathrm{F}^{-}$(green) ion as a function from the surface. Each curve presents the density of the water molecules on the half unit cell area $(0.20 \mathrm{~nm} \times 0.20$ $\mathrm{nm})$. 


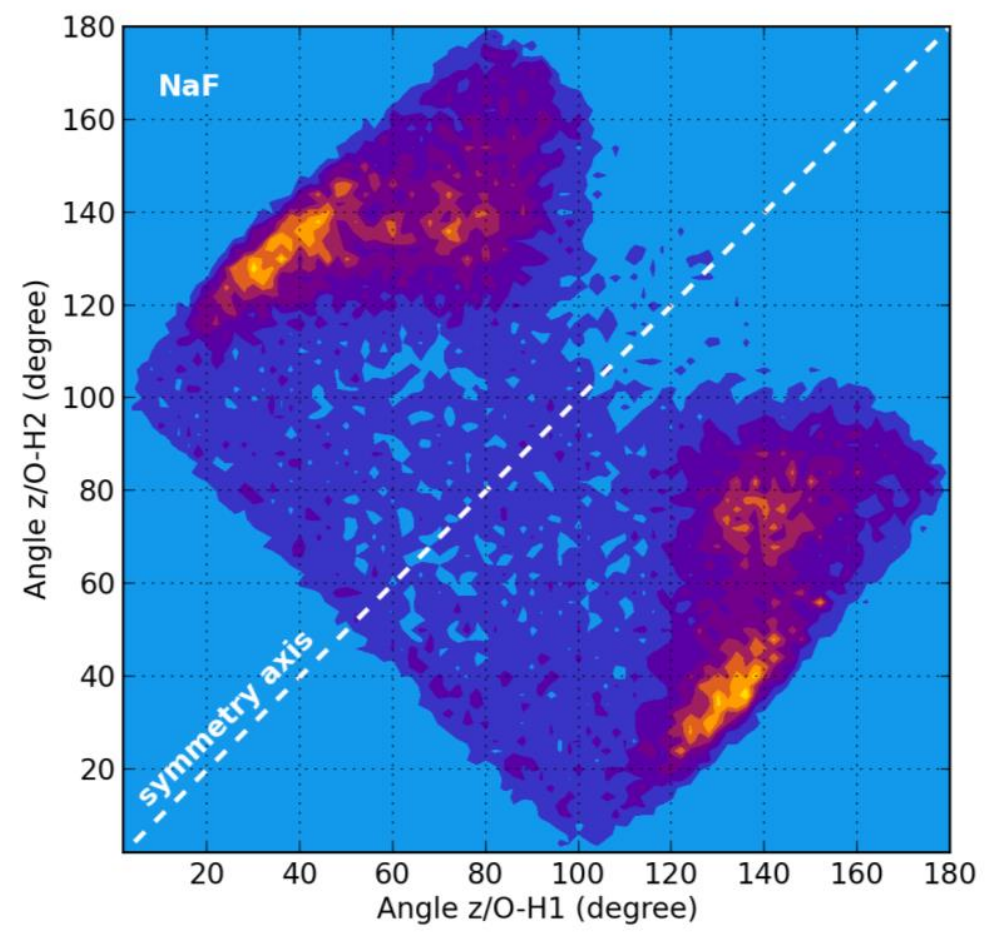

(a)

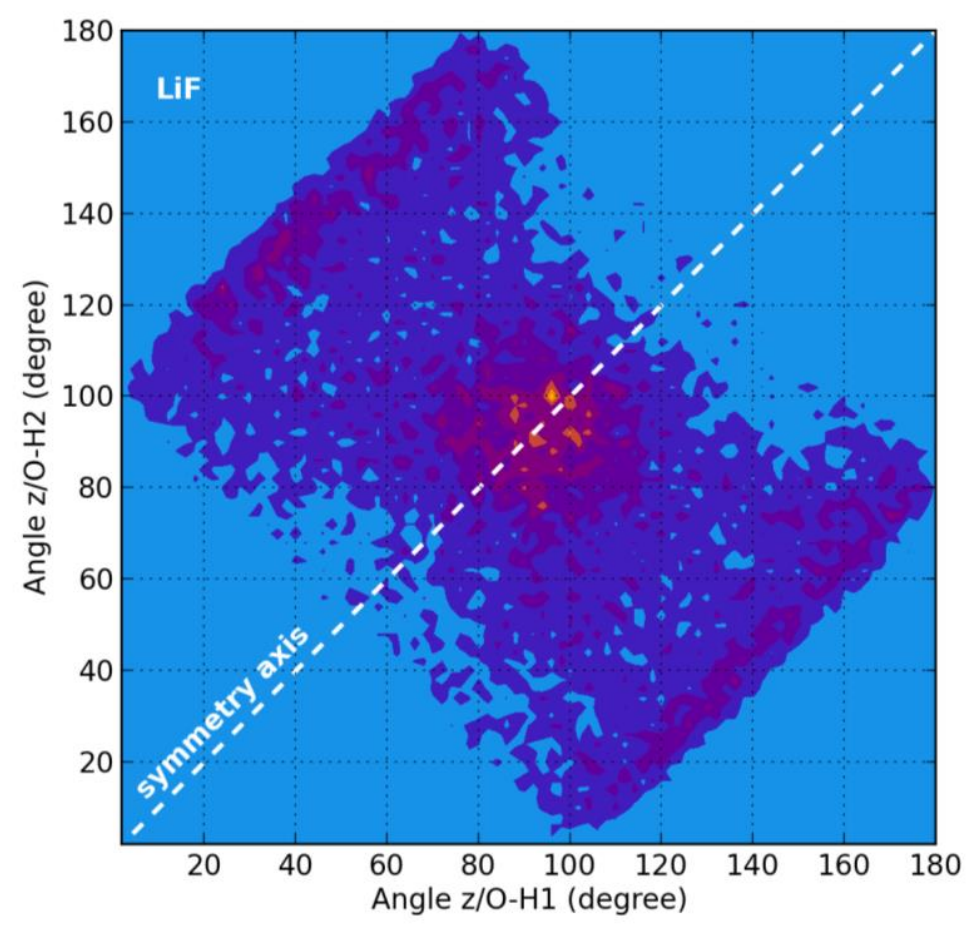

(b)

Figure S7: 2D correlation map of the angles of either OH-bond of the water molecules with respect to the surface normal. As both $\mathrm{OH}$-bonds are indistinguishable there is a symmetry around the diagonal. The lighter the color the stronger the correlation. In (a) the correlation for NaF is shown and in (b) for LiF. 